\title{
Functionalization with TAT-Peptide Enhances Blood-Brain Barrier Crossing In vitro of Nanoliposomes Carrying a Curcumin-Derivative to Bind Amyloid- $\beta$ Peptide
}

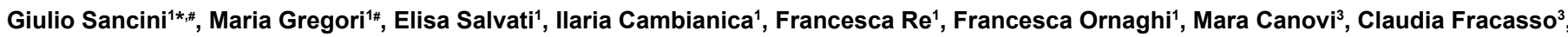
Alfredo Cagnotto ${ }^{3}$, Miriam Colombo ${ }^{2}$, Cristiano Zona ${ }^{2}$, Marco Gobbi ${ }^{3}$, Mario Salmona ${ }^{3}$, Barbara La Ferla ${ }^{2}$, Francesco Nicotra ${ }^{2}$ and Massimo $^{2}$ Masserini ${ }^{1}$

${ }^{1}$ Department of Health Sciences, University of Milano-Bicocca, via Cadore 48, 20900 Monza, MB, Italy

${ }^{2}$ Department of Biotechnology and Biosciences, University of Milano-Bicocca, Piazza della Scienza 2, 20126 Milano, Italy

${ }^{3}$ Department of Molecular Biochemistry and Pharmacology, Mario Negri Institute for Pharmacological Research, Via La Masa 19, 20156 Milano, Italy

\#These authors contributed equally to this work

\begin{abstract}
Production of abnormally high amounts of amyloid- $\beta$ peptide in the brain plays a central role in the onset and development of Alzheimer's disease, a neurodegenerative disorder affecting millions of individuals worldwide. Nanoparticles have been proposed as promising tools to treat the disease by delivering drugs and contrast agents to the brain. Here, nanoliposomes decorated with a curcumin-derivative, displaying high affinity for amyloid- $\beta$, were functionalized with a modified cell-penetrating TAT-peptide, with the aim of conferring on such nanoliposomes the ability to cross the blood-brain barrier. Functionalization with TAT-peptide did not modify the ability of curcumindecorated nanoliposomes to bind amyloid- $\beta$ fibrils, as assessed by surface plasmon resonance. Confocal microscopy, mass spectrometry and radioactivity experiments with $\left[{ }^{3} \mathrm{H}\right]$-sphingomyelin showed about 3 -fold increase in the uptake of nanoliposomes by human brain capillary endothelial cells (hCMEC/D3) after the functionalization with TATpeptide, with no alterations in cell viability. Moreover, TAT functionalization increased the permeability of curcuminnanoliposomes across a blood-brain barrier model made with the same cells. The similar permeabilities of curcuminderivative and $\left[{ }^{3} \mathrm{H}\right]$-sphingomyelin suggested that nanoliposomes were transported intact. Considering these results, nanoliposomes functionalized with the curcumin-derivative and TAT-peptide represent a promising tool for targeting amyloid- $\beta$ directly in the brain parenchyma.
\end{abstract}

Keywords: Alzheimer disease (AD); Amyloid $\beta(\mathrm{A} \beta)$; Curcumin; TAT-peptide; Liposomes; Blood-Brain barrier (BBB)

\section{Introduction}

Alzheimer's Disease (AD) is the most common neurodegenerative disease in the elderly population [1-3]. The mechanisms underlying AD are not yet completely clear but genetic, pathological and biochemical clues suggest that the progressive production and subsequent accumulation of the amyloid- $\beta(A \beta)$ peptides play a central role. Under abnormal conditions, the accumulation of $A \beta$ progressively forms oligomeric, multimeric and fibrillar aggregates and culminates with the formation of extracellular plaques, one of the morphological hallmarks of the disease [4-6].

In the search of new strategies for $\mathrm{AD}$ treatment, molecules able to stabilize the soluble $A \beta$ conformation, to destabilize the altered amyloidogenic conformer, and to prevent its aggregation could be effective inhibitors of amyloid plaque formation and very potent drug candidates [7-10]. Among them, curcumin (1, 1-diferuloylmethane) is a natural molecule with a wide variety of biological effects $[11,12]$. Curcumin is an active principle of the perennial herb Curcuma longa (commonly known as turmeric) which is used in the Indian traditional diet and as herbal medicine [13]. Recent studies indicate a role for curcumin as a potential anti-amyloid agent in vitro [14-16] and in vivo $[17,18]$. However, curcumin is unstable in aqueous media [19] and presents low bioavailability following delivery through oral or parenteral route [20]. The attachment of curcumin on the surface of Nanoparticles (NP) might increase the drug bioavailability [21]. Moreover, NP offer an attractive tool in driving drugs to the brain, due to their high potential for surface functionalization with ligands able to promote the crossing of the Blood-Brain Barrier (BBB) [2224], a tightly packed layer of endothelial cells surrounding the brain preventing high-molecular weight molecules from passing through [25]. Indeed, the design and engineering of NP with high specificity for brain capillary endothelial cells have been proposed as promising strategy for $\mathrm{AD}$ diagnosis and treatment [26-29].

The aim of the present investigation was to design NP able to bind $\mathrm{A} \beta$ peptide and to cross the BBB. To reach this goal we developed nanoliposomes (NL) double-functionalized with a curcuminderivative and with a modified HIV Transactivating Transcriptional Activator (TAT) peptide. Preparation of NL covalently decorated with curcumin-derivative was previously carried out, starting from a curcumin alkyne-derivative showing a very high affinity for $A \beta$ peptide [30], suitable for NL decoration by click chemistry and with improved features of stability with respect to curcumin itself [31]. On the other side, TAT-peptide could enhance NP BBB crossing [32,33] based on the evidence that its coupling to NP may facilitate their efficient translocation through the cell membrane, bypassing the endocytic pathway [34-36]. TAT-peptide was covalently attached to NL surface via a thiol-maleimide reaction. The ability of NL to bind $A \beta$ after

*Corresponding author: Giulio Sancini, University of Milano-Bicocca, Department of Health Sciences, via Cadore 48, 20900, Monza, Italy, Tel: 39-0264488310; Fax: 39-0264488068; E-mail: giulio.sancini@unimib.it

Received March 07, 2013; Accepted April 02, 2013; Published April 05, 2013

Citation: Sancini G, Gregori M, Salvati E, Cambianica I, Re F, et al. (2013) Functionalization with TAT-Peptide Enhances Blood-Brain Barrier Crossing In vitro of Nanoliposomes Carrying a Curcumin-Derivative to Bind Amyloid- $\beta$ Peptide. J Nanomed Nanotechol 4: 171. doi:10.4172/2157-7439.1000171

Copyright: ( 2013 Sancini G, et al. This is an open-access article distributed under the terms of the Creative Commons Attribution License, which permits unrestricted use, distribution, and reproduction in any medium, provided the original author and source are credited. 
Citation: Sancini G, Gregori M, Salvati E, Cambianica I, Re F, et al. (2013) Functionalization with TAT-Peptide Enhances Blood-Brain Barrier Crossing In vitro of Nanoliposomes Carrying a Curcumin-Derivative to Bind Amyloid- $\beta$ Peptide. J Nanomed Nanotechol 4: 171. doi:10.4172/21577439.1000171

TAT functionalization was assessed by surface plasmon resonance technique. The BBB crossing was investigated by measuring the NL permeability across a transwell BBB cellular model, made by human cerebral microvascular (hCMEC/D3) cultured cells, following $\left[{ }^{3} \mathrm{H}\right]-$ sphingomyelin added to nanoliposome as tracer by means of liquid scintillation counting and curcumin-derivative by mass spectrometry.

\section{Materials and Methods}

\section{Materials}

All chemical reagents were from Sigma-Aldrich (Milano, Italy). Bovine brain sphingomyelin (Sm), cholesterol (Chol), 1, 2-distearoylsn-glycero-3-phosphoethanolamine- $\mathrm{N}$-[maleimide(polyethylene glycol)-2000] (mal-PEG-DSPE) were purchased from Avanti Polar Lipids Inc., (Alabaster, USA). N-(4, 4-difluoro-5, 7-dimethyl-4-bora3a, 4a-diaza-s-indacene-3-dodecanoyl) sphingosyl phosphocholine (fluorescently labelled sphingomyelin, BODIPY-Sm) was from Molecular Probes (Milano, Italy). [ $\left.{ }^{3} \mathrm{H}\right]-\mathrm{Sm}$ and Ultima Gold were from Perkin Elmer (Waltham, USA). PD-10 columns were purchased from GE Healthcare (Uppsala, Sweden). A $\beta_{1-42}$ peptide, Triton X-100 and Sepharose CL-4B were purchased from Sigma-Aldrich (Milano, Italy). Amicon Ultra-15 centrifugal $10 \mathrm{~K}$ filter devices and polycarbonate filters for extrusion procedure were purchased from Millipore Corp (Bedford, USA). Extruder was from Lipex Biomembranes (Vancouver, Canada). NOVASYN-TGA resin was from Novabiochem (Darmstadt, Germany). Fmoc-protected L-amino acids were from Flamma (Bergamo, Italy). EBM-2 medium was from Lonza (Basel, Switzerland). Rat type I collagen, 1/100 chemically defined lipid concentrated, phalloydin and all the media and supplements for cell cultures were from Invitrogen Srl (Milano). LAMP-1 was from Abcam (Cambridge, UK). EEA1 was from BD (Becton Drive, Franklin Lakes, NJ, USA). Transwell permeable supports $0.4 \mu \mathrm{m}$ polyester membrane $12 \mathrm{~mm}$ insert, 12 well plates were from Corning (NY, USA). The hCMEC/D3 cell line was obtained under license from Institut National de la Santé et de la Recherche Medicale (INSERM, Paris, France).

\section{Assessment of Curcumin-derivative stability}

A curcumin-derivative with a terminal alkyne group ( $N$-propargyl 2-(3', 5'-di(4-hydroxy-3-metoxystyryl)-1H-pyrazol-1 ' -yl)-acetamide) (Curc) was synthesized as previously described [31]. $6 \mathrm{mg} / \mathrm{mL}$ of Curc were dissolved in acetone and the fluorescence spectrum was recorded $\left(\lambda_{\text {ex }}=340 \mathrm{~nm}\right)$ after different times of incubation (0-180 min) at $25^{\circ} \mathrm{C}$, using Cary Eclipse spectrofluorimeter (Varian). The spectra were compared with $6 \mathrm{mg} / \mathrm{mL}$ of native curcumin dissolved in acetone $\left(\lambda_{\mathrm{ex}}=420 \mathrm{~nm}\right)$ [37].

\section{TAT-peptide synthesis and characterization}

The sequence corresponding to residues 48-57 of human TAT protein was synthesized on an automated Applied Biosystems synthesizer model 433A (Applied Biosystems, Foster City, California) at $0.1 \mathrm{mM}$ scale with NOVASYN-TGA resin, using Fmoc-protected $\mathrm{L}$-amino acids. The peptides were bearing at the $C$-terminal a tryptophan residue for monitoring the peptide by fluorescence and ended with cysteine residue for covalent coupling with mal-PEGDSPE. Amino acids were activated by reaction with $O$-(Benzotriazol1-yl)-N,N,N',N'-tetramethyluronium tetrafluoroborate and $N, N$ diisopropylethylamine. A capping step with acetic anhydride after the last coupling cycle of each amino acid was included. Peptides were cleaved from the resin with trifluoroacetic acid/water/3, 6-dioxa-1, 8-octanedithiol (90:5:5 vol/vol/vol, $180 \mathrm{~min}$ at $\left.25^{\circ} \mathrm{C}\right)$, precipitated, and washed with diethyl ether. Crude peptide was then purified by reverse-phase high-performance liquid chromatography on a semipreparative C18 column (Symmetry 300; Waters Corporation, Milford, Massachusetts), and peaks collected were characterized by matrix assisted laser desorption/ionization mass spectrometry (MALDI-TOF). The sequence was GRKKRRQRRRPPQGWC (2065.47 $\mathrm{g} / \mathrm{mol}$ ) and purity was higher than $95 \%$.

\section{NL preparation}

NL were composed of a matrix of $\mathrm{Sm} /$ Chol (1:1 molar ratio) mixed with 10 molar\% of a PEGylated lipid containing an azido terminus (3-deoxy-1, 2-dipalmitoyl-3-(4'-methyl(O-(2-azidoethyl)heptaethylenglycol-2-yl)-ethylcarbamoylmethoxy ethylcarbamoyl-1H1',2',3'-triazol-1' -yl)-sn-glycerol) (azido-PEG-lipid), for the coupling with Curc, synthesized according to a previous publication [30], and 2.5 molar \% of mal-PEG-DSPE, for the coupling with TAT-peptide. For preparation of fluorescently labelled NL, 0.5 molar $\%$ of total Sm was substituted with BODIPY-Sm. For uptake and permeability studies, 0.001 molar $\%$ of $\left[{ }^{3} \mathrm{H}\right]-\mathrm{Sm}$ was added as tracer to follow lipid distribution by radioactivity counting. NL was prepared in $10 \mathrm{mM}$ PBS, $\mathrm{pH} 7.4$ by extrusion procedure through a $100-\mathrm{nm}$ pores polycarbonate filter, as previously described [38]. Phospholipid recovery after extrusion was determined by phosphorous assay using the method of Stewart [39].

\section{Preparation of Curc-decorated nanoliposomes (Curc-NL) by 'click chemistry'}

Click reaction between azido-PEG-lipid containing NL and Curc was performed using the Huisgen 1, 3-dipolar cycloaddition of azides and terminal alkynes, with some modification from [30]. Briefly, an aqueous solution of $\mathrm{CuSO}_{4}(8 \mathrm{mM})$ and sodium ascorbate $(145 \mathrm{mM})$ were added to a solution of Curc (100 mM in DMSO). The obtained mixture was added to NL and the reaction was gently stirred for $6 \mathrm{~h}, \mathrm{pH}$ 6.5 , at $25^{\circ} \mathrm{C}$. The resulting mixture was purified by ultrafiltration with Amicon Ultra-15 devices and by gel filtration through a Sepharose 4BCL column $(25 \times 1 \mathrm{~cm})$. NL elution was assessed by DLS (Dynamic Light Scattering). The presence of Curc linked to lipid (Curc-PEG-lipid) in NL after purification was verified by mass spectrometry (see below). Phospholipid recovery was determined as described above [39].

\section{Functionalization of Curc-NL with TAT-peptide (TAT-Curc- NL)}

TAT-peptide was added to maleimide containing Curc-NL in PBS to give a final peptide-to-maleimide molar ratio of 1.2:1. The mixture was incubated overnight at $25^{\circ} \mathrm{C}$. Peptide-bound $\mathrm{NL}$ was separated from the unbound peptide using a PD-10 column. The yield of coupling and the amount of coupled peptide was assessed by tryptophan fluorescence intensity measurements, as reported [40]

\section{Characterization of NL}

All NL preparations were characterized in terms of size, $\zeta$-potential, polydispersity index and stability by DLS, as described [38]

\section{HPLC-MS/MS method for Curc-PEG-lipid quantification}

The HPLC system consisted of an Alliance separation module 2695 (Waters, Milliford, MA, USA) and a chromatographic X Terra C8 column $3.5 \mu \mathrm{m}, 150 \times 2.0 \mathrm{~mm}$ (Waters, Milliford, MA, USA) coupled with a security guard C8 cartridges $2.1 \times 10 \mathrm{~mm}$ (Waters, Milliford, MA, USA), held at $30^{\circ} \mathrm{C}$. The HPLC system was coupled with a Micromass Quattro Micro triple-quadrupole mass spectrometer (MS, Waters, Milliford, MA, USA), controlled by Mass-Lynx software 4.0 
Citation: Sancini G, Gregori M, Salvati E, Cambianica I, Re F, et al. (2013) Functionalization with TAT-Peptide Enhances Blood-Brain Barrier Crossing In vitro of Nanoliposomes Carrying a Curcumin-Derivative to Bind Amyloid- $\beta$ Peptide. J Nanomed Nanotechol 4: 171. doi:10.4172/21577439.1000171

Page 3 of 8

and equipped with an electrospray ionization interface using argon as collision gas. MS analyses were done using positive ionization and multiple reactions monitoring mode, measuring the fragmentation products of the deprotonated pseudo-molecular ions of Curc-PEGlipid. The choice of fragmentation products and the optimization of collision-induced dissociation energies and other instrumental parameters were done in continuous-flow mode, using Curc-PEG-lipid solutions ( $50 \%$ methanol containing $0.05 \%$ formic acid) as standard. Samples were analyzed with the ion spray needle operating at $4.0 \mathrm{kV}$, cone voltage at $43 \mathrm{~V}$, collision energy at $30 \mathrm{eV}$, source and desolvation temperatures at 100 and $300^{\circ} \mathrm{C}$, respectively. The $\mathrm{m} / \mathrm{Z}$ of the bicharged Curc-PEG-lipid ion was 823.0 and the principal ion transition 823.0 $>377.3$ were selected for quantification. The Mobile Phases (MP) were composed by: $0.05 \%$ formic acid in acetonitrile/water, 80:20 (MPA), and $0.05 \%$ formic acid in acetonitrile (MP-B). The HPLC system was set up to operate at a flow rate of $0.2 \mathrm{~mL} / \mathrm{min}$ (from 0 to $100 \%$ of solvent B in $10 \mathrm{~min}$, hold at $100 \%$ for $10 \mathrm{~min}$ and re-equilibration for $6 \mathrm{~min}$ at $0 \%$ of solvent B). The retention time for Curc-PEG-lipid was 11.9 min.

\section{Interaction of $\mathrm{NL}$ with $\mathrm{A} \beta$ investigated by Surface Plasmon Resonance (SPR)}

SPR experiments were conducted by using a SensiQ semi-automatic SPR machine (ICx Technologies) with two parallel flow channels; one was used to immobilize $A \beta$ fibrils while the other was used as "reference" (empty surface). A $\beta$ aggregates were prepared as already described [38] and immobilized on a COOH5 sensor chip (ICx Technologies) by amine coupling chemistry using the same procedure described by Le Droumaguet et al. [41]. The final immobilization level was $\sim 5000$ Response Unit $\left(\mathrm{RU}, 1 \mathrm{RU}=1 \mathrm{pg} / \mathrm{mm}^{2}\right)$. The empty "reference" surface was prepared following the same immobilization procedure, without the addition of the peptide. Sensorgrams were obtained via subsequent injections of not-functionalized NL and TAT-Curc-NL flowed at 30 $\mu \mathrm{L} / \mathrm{min}$ for $5 \mathrm{~min}$, at three different concentrations $(2,6,12 \mu \mathrm{M}$, of total lipids) over the immobilized ligand or the reference surface, at the same time. The non-specific binding to the reference channel was automatically subtracted from the total signal. The binding profiles were processed by Qdat Software (ICx Technologies) and a "global" fit of the entire series of curves, obtained according to the pseudofirst order 1:1 Langmuir interaction model and according to the same model incorporating mass transport limitation, was used to calculate association $\left(\mathrm{K}_{\mathrm{a}}\right)$ and dissociation $\left(\mathrm{K}_{\mathrm{d}}\right)$ rate constant and the corresponding $\mathrm{KD}$.

\section{Culture of hCMEC/D3 cells}

hCMEC/D3 cells (passage 25-35) were seeded at a concentration of 27000 cells $/ \mathrm{cm}^{2}$ and grown in tissue culture flasks coated with $0.1 \mathrm{mg} /$ $\mathrm{mL}$ rat tail collagen type 1, in EBM-2 medium supplemented with $5 \%$ fetal bovine serum, $1 \%$ Penicillin-Streptomycin, $1.4 \mu \mathrm{M}$ hydrocortisone, $5 \mu \mathrm{g} / \mathrm{mL}$ ascorbic acid, 1/100 chemically defined lipid concentrate 10 $\mathrm{mM}$ HEPES and $1 \mathrm{ng} / \mathrm{mL}$ basic fibroblast growth factor. The cells were cultured at $37^{\circ} \mathrm{C}, 5 \% \mathrm{CO}_{2} /$ saturated humidity. Cells culture medium was changed every 2-3 days.

\section{Assessment of NL cytotoxicity on hCMEC/D3 cells}

hCMEC/D3 cells $\left(30000\right.$ cells $\left./ \mathrm{cm}^{2}\right)$ were grown on 12 -well plates coated with type I collagen. Medium was replaced and NL $(100 \mu \mathrm{M})$ suspended in cell culture medium were incubated at $37^{\circ} \mathrm{C}$ with the cells for up to $48 \mathrm{~h}$, corresponding to 2 -fold the cell doubling time. After treatment, the cell viability was assessed by MTT (3-(4, 5-dimetiltiazol2-yl)-2, 5-diphenyltetrazolium bromide) assay, as described [40]. Each sample was analyzed at least in triplicate.

\section{NL uptake studies carried out by confocal-laser-scanning microscopy (CLSM)}

CLSM was employed in order to study the cellular uptake of fluorescently labelled NL. Pictures were taken using an LSM710 inverted CLSM equipped with a Plan-Neofluar 63×/1.4 oil objective (Carl Zeiss, Oberkochen, Germany). Excitation was performed using two V/VISlaser diode $25 \mathrm{mV}(405-488)$ and Ar-laser $(540 \mathrm{~nm})$ at $10 \%$ intensity. The pinhole was set to 1 Airy. Image acquisition was done sequentially to minimize cross-talk between the fluorophores. $30000 / \mathrm{cm}^{2}$ cells were cultured for 2 days on rat type I collagen-coated cover slips (diameter $25 \mathrm{~mm}$ ) positioned in culture dishes, and incubated at $37^{\circ} \mathrm{C}$ with CurcNL or TAT-Curc-NL containing BODIPY-Sm suspended in serum free medium for $3 \mathrm{~h}$, rinsed 3 times with PBS and fixed with a 10\% formalin solution. NL design has been optimized to achieve recognition by the targeted cells, and approaches such as the one here presented can be used to determine whether targeting is indeed achieved. All the results presented from now on were obtained in serum free medium. Before moving towards in vivo studies, we will verify whether NL targeting is preserved in more realistic biological environments. After 3 washes with PBS, cells were permeabilized with $0.2 \%$ Triton-X100 in PBS for $15 \mathrm{~min}$, then rinsed twice and incubated with a solution of $1 \%$ phalloydin (actin filaments staining) in PBS for $1 \mathrm{~h}$, then with $20 \mu \mathrm{M}$ DAPI (nuclear staining) in PBS for $10 \mathrm{~min}$ and finally with LAMP-1 (1:200) (late endosomes and early lysosomes staining) or EAA1 (1:200) (early endosomes staining), for $4 \mathrm{~h}$, at $25^{\circ} \mathrm{C}$. After 3 washes in PBS, the samples were mounted using polyvinyl alcohol mounting medium.

\section{NL uptake and permeability across hCMEC/D3 cell monolayer}

To quantify the association of NL with hCMEC/D3 cells and the permeability across a monolayer made with the same cells, two NL components, $\left[{ }^{3} \mathrm{H}\right]-\mathrm{Sm}$ and Curc-PEG-lipid, were followed by liquid scintillation counting and MS, respectively.

For uptake experiments, NL containing $\left[{ }^{3} \mathrm{H}\right]-\mathrm{Sm}$ as tracer were suspended in serum free culture medium at $100 \mu \mathrm{M}$ of total lipids concentration, added to hCMEC/D3 cells $\left(30000\right.$ cells $\left./ \mathrm{cm}^{2}\right)$ grown in 12 -well plates, and incubated for $3 \mathrm{~h}$ at $37^{\circ} \mathrm{C}$. The medium was withdrawn and cells were washed with $1 \mathrm{~mL}$ culture medium containing $0.5 \%$ serum, $1 \mathrm{~mL}$ PBS and with $400 \mu \mathrm{L}$ trypsin $0.1 \%$ for $3 \mathrm{~min}$, followed by PBS washing. Finally, the cells were detached with $400 \mu \mathrm{L}$ trypsin $0.25 \% /$ EDTA. The suspensions were mixed with $5.0 \mathrm{~mL}$ Ultima Gold scintillation cocktail and counted with a Tri-Carb 2200 CA Liquid Scintillation Analyzer (Packard) for $\left[{ }^{3} \mathrm{H}\right]-S m$ quantification. To quantify Curc-PEG-lipid by MS, samples were extracted with 4 volumes of ethyl-acetate; after shaking for $15 \mathrm{~min}$, samples were centrifuged for $2 \mathrm{~min}$ at $1000 \mathrm{rpm}$, the solvent layer recovered and dried under nitrogen at $25^{\circ} \mathrm{C}$. The residues were then dissolved in 100 $\mu \mathrm{L}$ MP-A and $20 \mu \mathrm{L}$ were injected into the HPLC system at $10^{\circ} \mathrm{C}$. A calibration curve was prepared containing known amounts of CurcPEG-lipid (linearity range from 0.5 to $300 \mathrm{ng}$ ), and then extracted using the same procedure. Peak area of Curc-PEG-lipid was used for quantification by MS.

For permeability experiments across a cell monolayer, hCMEC were seeded in a 12-well transwell inserts coated with type I collagen. $0.5 \mathrm{~mL}$ of cell suspensions containing $6 \times 10^{4}$ cells were added to the upper (donor) chamber which was inserted into the lower (acceptor) chamber containing $1 \mathrm{~mL}$ of the culture medium. A tight cell monolayer was usually formed 8-10 days after seeding, judged by three criteria: (1) the cells formed a confluent monolayer without visible spaces between cells under a light microscope; (2) the height of the culture 
Citation: Sancini G, Gregori M, Salvati E, Cambianica I, Re F, et al. (2013) Functionalization with TAT-Peptide Enhances Blood-Brain Barrier Crossing In vitro of Nanoliposomes Carrying a Curcumin-Derivative to Bind Amyloid- $\beta$ Peptide. J Nanomed Nanotechol 4: 171. doi:10.4172/21577439.1000171

Page 4 of 8

medium in the upper chamber had to be at least $2 \mathrm{~mm}$ higher than that in the lower chamber for at least $24 \mathrm{~h}$; and (3) a constant TEER (Trans Endothelial Electrical Resistance) value, measured using an EVOM Endohm chamber (World Precision Instruments, Sarasota, FL) was obtained. Wells were used when TEER value was higher than 40 $\Omega \cdot \mathrm{cm}^{2}$. Transendothelial permeability coefficient $(\mathrm{PE})$ was calculated as previously described [40]. All the permeability experiments were performed in serum free culture medium at $37^{\circ} \mathrm{C}$, adding $100 \mu \mathrm{M}$ (as lipids) NL incorporating about $20000 \mathrm{dpm} / \mathrm{ml}$ of $\left[{ }^{3} \mathrm{H}\right]-\mathrm{Sm}$ into the upper chamber. After adding NL to the upper compartment, aliquots were taken from the lower compartment at different times (0-60$180 \mathrm{~min}$ ) for liquid scintillation counting and for Curc-PEG-lipid quantification, as above described. In parallel experiments, the efflux of the hydrophilic marker $\left[{ }^{14} \mathrm{C}\right]$-sucrose $(200 \mu \mathrm{M})$ added in the upper chamber was measured to evaluate the paracellular permeability. To monitor cell monolayer integrity during permeability studies, we performed co-incubation with unlabelled $\mathrm{NL}$ and $\left[{ }^{14} \mathrm{C}\right]$-sucrose. At $0,60,180 \mathrm{~min}\left[{ }^{14} \mathrm{C}\right]$-sucrose radioactivity was counted and PE was calculated as described [40].

\section{Statistical analysis}

Each experiment was performed at least in triplicate. The differences were evaluated for statistical significance using Student's $t$-test.

\section{Results}

\section{Time-dependent stability of Curc}

Fluorescence spectra of Curc and native curcumin were recorded after incubation for different times. The results showed that the maximum fluorescence intensity of native curcumin was reduced of more than $50 \%$ in the first $2 \mathrm{~h}$ of incubation, compared with a reduction of only $10 \%$ for Curc. Moreover, Curc fluorescence was also detectable after longer incubation times (Figure 1).

\section{Curc-NL and TAT-Curc-NL preparation and characterization}

Curc was coupled to NL through a 'click reaction' between its triple bond and the azido function of azido-PEG-lipid, present in NL as 10 molar \% of total lipids. Theoretical structure of Curc-NL is depicted in figure 2. The yield of the 'click reaction' ranged between 75 and 95\%, as determined by quantifying Curc-PEG-lipid amount in Curc-NL dispersions by mass spectrometry (Figure $3 \mathrm{~A}$ ). Curc-NL preparations resulted monodispersed, with a mean diameter of $179.7 \pm 3.5 \mathrm{~nm}$ and negatively charged, as reported in table 1 .

TAT-peptide was coupled to Curc-NL through reaction between its cysteine thiol group and maleimide function onto the distal end of PEG chains of mal-PEG-DSPE lipid. After purification of TAT-CurcNL from the unbound peptide by gel filtration, fluorescence emission spectrum was recorded to verify the presence of TAT-peptide onto NL. The tryptophan fluorescence spectrum of TAT-Curc-NL displayed a blue shift to $350 \mathrm{~nm}$ in the emission maximum, with respect to 354 nm of TAT-peptide alone (Figure 3B), suggesting that the coupling of TAT with the mal-PEG-DSPE lipid occurred, as previously reported for other peptides [42]. The yield of coupling was between 60 and $70 \%$. After the coupling with TAT-peptide, TAT-Curc-NL preparations are still monodispersed, with slight modifications in size and charge (Table 1). The size of all NL preparations remained unchanged for at least 48 $\mathrm{h}$ (data not shown).

\section{SPR analysis of NL binding to $A \beta$ peptide}

SPR was exploited to investigate the association and dissociation of
NL to A $\beta$ fibrils (Figure 4). TAT-Curc-NL, as well as not functionalized $\mathrm{NL}$, was injected at concentration of $2,6,12 \mu \mathrm{M}$ total lipids over parallel flow channels of the same sensor chip with immobilized $A \beta$ fibrils or nothing (reference surface). The non-specific binding to the reference channel was automatically subtracted from the total signal. Only negligible binding was detected for not functionalized NL (Figure $4 \mathrm{~A})$; on the contrary, TAT-Curc-NL interacted with immobilized A $\beta$ as indicated by the marked increase of RU signal versus time (Figure 4B). The "global" fit of the entire series of curves was obtained according to the pseudo-first order 1:1 Langmuir interaction model. Fitting was significantly better by incorporating mass transport limitation, and are shown as grey lines in figure $4 \mathrm{~B}$. The $\mathrm{KD}$ value of TAT-Curc-NL towards $A \beta$ calculated on total lipids concentration was in the low nanomolar range: $\mathrm{KD}=48 \mathrm{nM}$.

\section{Assessment of NL cytotoxicity on hCMEC/D3 cells}

NL toxicity was tested using MTT assay. All the NL preparations tested were non-toxic, since the cell viability of hCMEC/D3 cells
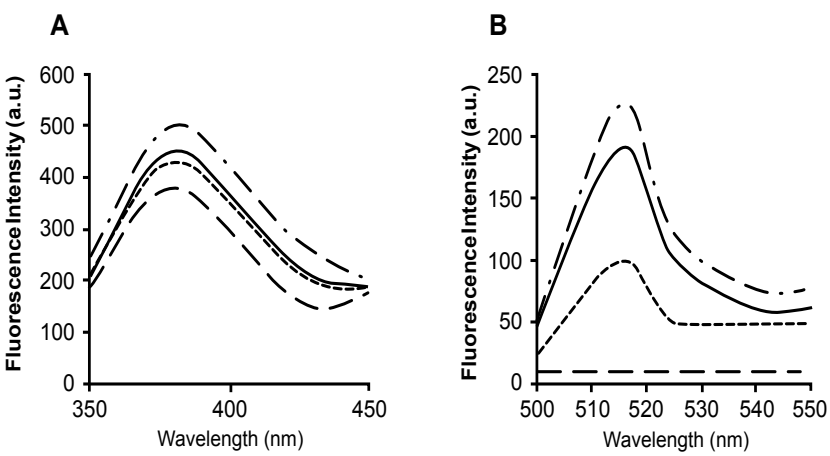

Figure 1: Time-dependent stability of Curcumin-derivative (Curc). Curc (A) or native curcumin (B) were dissolved in acetone $(6 \mathrm{mg} / \mathrm{mL})$ and the fluorescence spectra were recorded at different times $(0, \cdot-\cdot 60,-, 120$, $---, 180 \mathrm{~min},--)$ at $25^{\circ} \mathrm{C}$, with $\lambda_{\text {ex }}$ of $340 \mathrm{~nm}$ or $420 \mathrm{~nm}$ for Curc or curcumin, respectively.
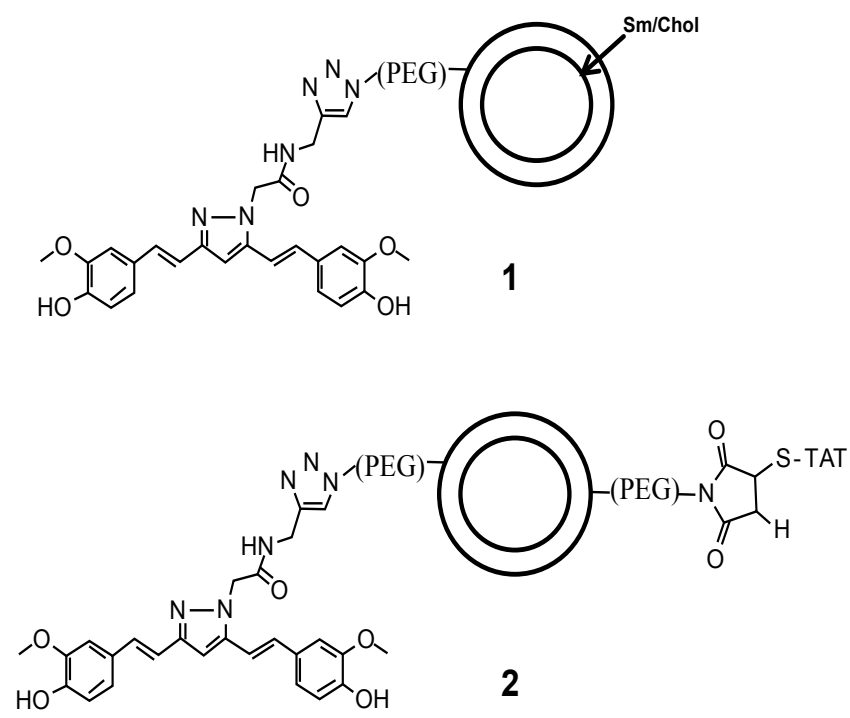

Figure 2: NL structure: Theoretical structure of Curc-NL (1) and TAT-Curc$\mathrm{NL}$ (2). Curc=curcumin-derivative; $\mathrm{NL}=$ nanoliposomes. 
Citation: Sancini G, Gregori M, Salvati E, Cambianica I, Re F, et al. (2013) Functionalization with TAT-Peptide Enhances Blood-Brain Barrier Crossing In vitro of Nanoliposomes Carrying a Curcumin-Derivative to Bind Amyloid- $\beta$ Peptide. J Nanomed Nanotechol 4: 171. doi:10.4172/21577439.1000171

Page 5 of 8

A
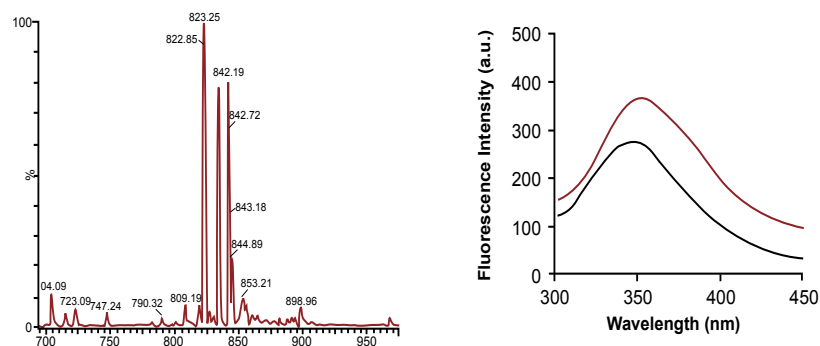

Figure 3: Characterization of Curc and TAT-peptide binding reactions on NL surface.

NL were functionalized with Curc and TAT-peptide and purified as described in the text.

A) Representative mass spectrometry spectrum of Curc-PEG-lipid, the product of the reaction between Curc and azido-PEG-lipid containing NL; B) Representative fluorescence spectra of TAT-peptide before (red) and after (black) conjugation to maleimide containing $\mathrm{NL}\left(\lambda_{\mathrm{ex}} 280 \mathrm{~nm}\right)$.

Curc=curcumin-derivative; $\mathrm{NL}=$ nanoliposomes

\begin{tabular}{|l|l|l|l|}
\hline Liposome type & Diameter \pm SD $(\mathbf{n m})$ & PI & $\zeta$-Potential \pm SD $(\mathbf{m V})$ \\
\hline Not functionalized NL & $112.5 \pm 1.2$ & 0.122 & $-20.87 \pm 1.09$ \\
\hline Curc-NL & $179.7 \pm 3.5$ & 0.176 & $-15.35 \pm 1.94$ \\
\hline TAT-Curc-NL & $196.5 \pm 3.2$ & 0.152 & $-12.94 \pm 0.94$ \\
\hline
\end{tabular}

$\mathrm{SD}=$ standard deviation; $\mathrm{Curc}=$ curcumin-derivative; $\mathrm{NL}=$ nanoliposomes

Table 1: Physicochemical properties of nanoliposomes (NL). Size distribution $(\mathrm{nm})$, polydispersity index $(\mathrm{PI})$ and $\zeta$-potential $(\mathrm{mV})$ are measured as described in the methods section. Values reported are the mean values from at least 5 measurements of 3 different preparations, in each case.

did not decrease below 96\% (data not shown) after $48 \mathrm{~h}$ incubation with NL. Moreover, after incubation with any NL formulation, the permeability of $\left[{ }^{14} \mathrm{C}\right]$-sucrose and TEER values did not change, within the experimental error $(<3 \%$ and $<1.5 \%$, respectively), thus suggesting no adverse effect on cell monolayer integrity (data not shown).

\section{Cellular uptake of NL studied by CLSM}

Cellular uptake by hCMEC/D3 cells of Curc-NL and TAT-CurcNL fluorescently-labelled with BODIPY-Sm was qualitatively evaluated by CLSM (Figure 5). In the case of Curc-NL very low amounts of fluorescence associated to cells were detected after $3 \mathrm{~h}$ incubation. On the contrary, the coupling of NL with TAT-peptide increased the amount of fluorescence associated to hCMEC/D3 cells. The images showed the presence of hot green spots below the plasma membrane and near the perinuclear recycling region.

Successively, we performed a staining of late-endosomes and earlylysosomes by means of LAMP-1 and early endosomes by means of EAA1. Our results show that Curc-NL and TAT-Curc-NL did not colocalize with early endosomes or late-endosomes and early-lysosomes, though their fluorescence was present in the same optical section (Figure 5), thus indicating their cellular uptake.

Cellular uptake and permeability investigated by radiochemical technique and mass spectrometry

We quantitatively evaluated the uptake of Curc-NL and TAT-CurcNL by hCMEC/D3 cells using $\left[{ }^{3} \mathrm{H}\right]$-Sm-labelling. After $3 \mathrm{~h}$ incubation with radiolabelled $\mathrm{NL}$, and mild trypsin treatment to remove membrane surface-adsorbed radioactivity, cells were detached with trypsin/EDTA solution and the radioactivity stably associated with cells was counted.
A

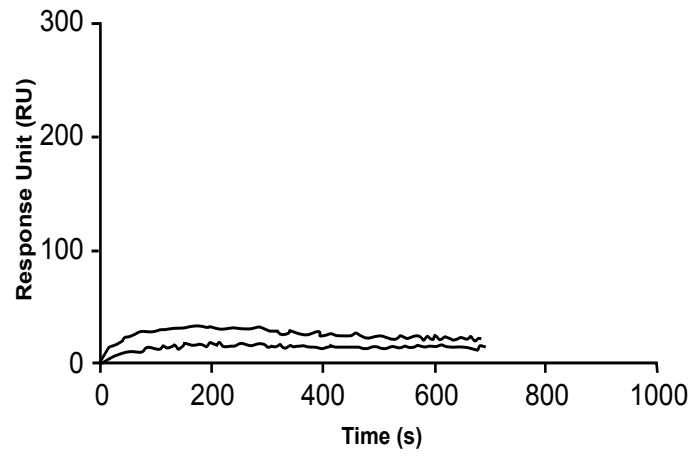

B

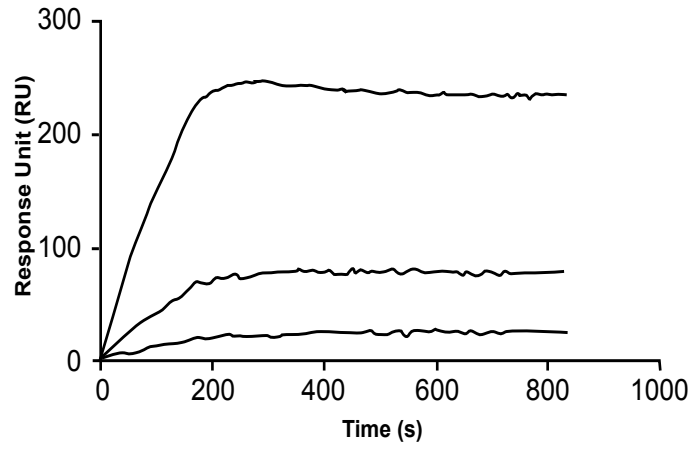

Figure 4: TAT-Curc-NL binding to $A \beta$ fibrils. Surface Plasmon Resonance sensorgrams resulting from the "global" fitting of the curves obtained from the interaction between $A \beta$ fibrils immobilized on a gold sensor surface and $A$ not functionalized NL or B) TAT-Curc-NL. NL were flowed in solution at total lipids concentrations of $2,6,12 \mu \mathrm{M}$ for $5 \mathrm{~min}$ at $30 \mu \mathrm{L} / \mathrm{min}$.

Curc=curcumin-derivative; $\mathrm{NL}=$ nanoliposomes.
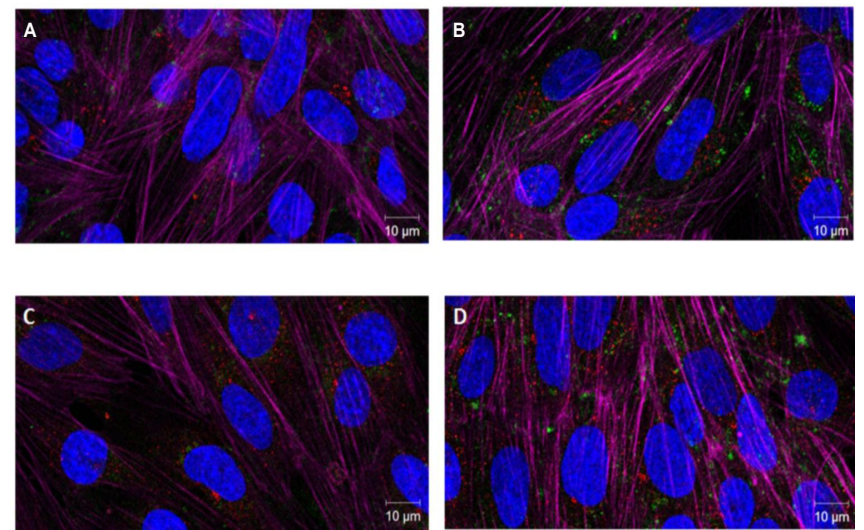

Figure 5: Uptake of Curc-NL and TAT-Curc-NL by hCMEC/D3 cell monolayers by CSLM. The localization and distribution of Curc-NL (A, C) and TAT-Curc-NL (B, D) fluorescently labelled with BODIPY-Sm (green fluorescence) within hCMEC/D3 cells. NL did not induce changes in actin staining in the cell monolayer. Fluorescent NL were visualized by CSLM: cells were incubated with Far red-Phalloidin to visualize the actin filaments (purple fluorescence), and nuclear staining was performed by DAPI (blue staining). Curc-NL displayed very low intracellular uptake (A, C). Curc-TAT-NL was more efficiently taken-up (B and D).

hCMEC/D3 cells were incubated with (A, B) LAMP-1 to mark late-endosomes and early-lysosomes and with (C, D) EAA1 to stain early endosomes (red staining). Neither Curc-NL nor Curc-TAT-NL co-localize with early endosomes and late endosomes/early-lysosomes. Scale bar=10 $\mu \mathrm{m}$. Curc=curcuminderivative; $\mathrm{NL}=$ nanoliposomes. 
Citation: Sancini G, Gregori M, Salvati E, Cambianica I, Re F, et al. (2013) Functionalization with TAT-Peptide Enhances Blood-Brain Barrier Crossing In vitro of Nanoliposomes Carrying a Curcumin-Derivative to Bind Amyloid- $\beta$ Peptide. J Nanomed Nanotechol 4: 171. doi:10.4172/21577439.1000171

Page 6 of 8

When hCMEC/D3 cells were incubated with Curc-NL and TAT-Curc$\mathrm{NL}$, the radioactivity stably associated with cells was $0.51 \pm 0.07 \%$ and $1.39 \pm 0.26 \%$ of the administered dose $(\mathrm{p}<0.05)$, respectively (Figure $6 \mathrm{~A})$.

Furthermore, the uptake of NL has been evaluated by quantification of Curc-PEG-lipid by HPLC-MS/MS. When hCMECD/3 cells were incubated with Curc-NL or TAT-Curc-NL, Curc stably associated with cells was $0.37 \pm 0.04 \%$ or $1.18 \pm 0,16 \%(\mathrm{p}<0.05)$ of the administered dose, respectively. These results proved that the cellular uptake of Curc-NL strongly increased after NL functionalization with TATpeptide. The $\zeta$-potential of the here prepared NL was slightly negative, nevertheless the TAT sequences added to NL surface has been found to drive a direct interaction of TAT-NL with hCMEC/D3 cells to sustain their greater intracellular uptake.

After 8-10 days in vitro, TEER values were constant in relation to time (typically $47.1 \pm 4.2 \Omega \times \mathrm{cm}^{2}$ ). At this time, the PE value for

A

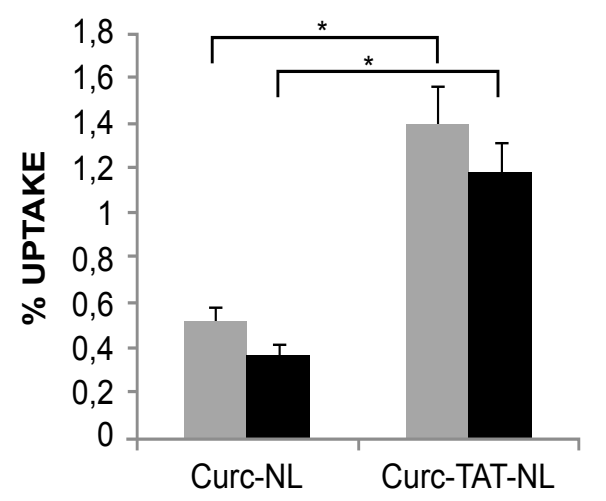

SM

- Curc

B

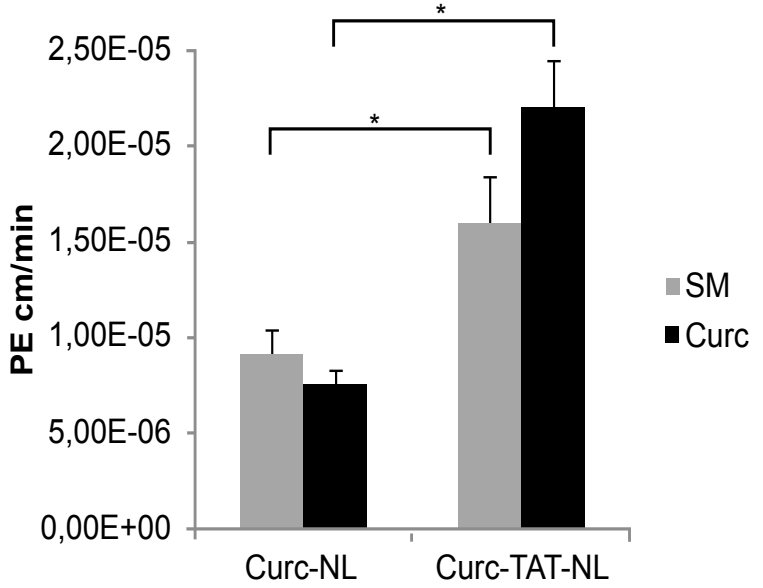

Figure 6: Uptake and permeability of Curc-NL and TAT-Curc-NL by hCMEC/D3 cell monolayers by radiochemical technique. $6 \times 10^{4}$ cells were incubated with $\mathrm{NL}$ labelled with $\left[{ }^{3} \mathrm{H}\right]-\mathrm{Sm}$ for $3 \mathrm{~h}$ at $37^{\circ} \mathrm{C}, 5 \% \mathrm{CO}_{2}$. Panel A. Uptake of NL. After incubation, the uptake of Curc-NL or TATCurc-NL by hCMEC/D3 cells has been assessed by following both tracers used, $\left[{ }^{3} \mathrm{H}\right]-\mathrm{Sm}$ (grey bars) by radioactivity counting, and Curc-PEG-lipid (black bars) by mass spectrometry. Panel B. Transcytosis of NL through hCMEC/D3 cell monolayers. The permeability (PE) of Curc-NL and TATCurc-NL across the cell monolayer was calculated for both tracers used, $\left[{ }^{3} \mathrm{H}\right]-\mathrm{Sm}$ (grey bars) and Curc-PEG-lipid (black bars). Each value is the mean of at least three independent experiments and the SDs of means are presented as bars. ${ }^{*}=p<0.05$. Curc $=$ curcumin-derivative; $\mathrm{NL}=$ nanoliposomes; $\mathrm{Sm}=$ sphingomyelin sucrose was in the order of $1.86 \pm 0.07 \times 10^{-3} \mathrm{~cm} / \mathrm{min}$. Accordingly; all subsequent permeability experiments were performed after 8-10 days in vitro. The PE value of Curc-NL, evaluated by counting $\left[{ }^{3} \mathrm{H}\right]-\mathrm{Sm}$ radioactivity $\left(9.06 \pm 1.3 \times 10^{-6} \mathrm{~cm} / \mathrm{min}\right)$, was enhanced in case of TATCurc-NL $\left(\mathrm{PE}=1.59 \pm 0.44 \times 10^{-5} \mathrm{~cm} / \mathrm{min}, \mathrm{p}<0.05\right)$ (Figure 6B).

Furthermore the PE has been evaluated assaying Curc-PEG-lipid by HPLC-MS/MS. The PE value $\left(7.5 \pm 0.075 \times 10^{-6} \mathrm{~cm} / \mathrm{min}\right)$ was enhanced in case of TAT-Curc-NL $\left(\mathrm{PE}=2.2 \pm 0.22 \times 10^{-5} \mathrm{~cm} / \mathrm{min}, \mathrm{p}<0.05\right)$ (Figure 6 ). The differences between uptake and $\mathrm{PE}$ values measured following $\left[{ }^{3} \mathrm{H}\right]-\mathrm{Sm}$ or Curc-PEG-lipid were comparable and not statistically significant (Figures 6A and 6B).

\section{Discussion}

With the number of people with dementia increasing rapidly worldwide, more than 36 million people with the disease today and more than 115 million predicted for the year 2050, care for people with dementia will put an unprecedented burden on health and social systems. Therefore, the search for effective therapies and early diagnosis, still lacking today, is imperative. So far, several therapeutic strategies have been proposed. Since extracellular aggregates (plaques) of the cytotoxic $\mathrm{A} \beta$ peptide in the $\mathrm{AD}$ brain are hallmarks of the disease, one therapeutic strategy considers $A \beta$ as target [43].

This study was aimed at the preparation and characterization of NL double-functionalized to bind Alzheimer's disease $A \beta$ peptide and to cross the BBB.

To bind $\mathrm{A} \beta$ peptide, NL functionalized with a curcuminderivative (Curc) with high affinity for $A \beta$ [30] were utilized. Curc has a good stability, as proved within the present investigation, much improved compared to curcumin itself, probably due to the absence of the chemically labile $\beta$-diketone moiety [31]. The coupling of Curc molecules to the surface of NL by 'click chemistry' proceeded easily and almost quantitatively at $25^{\circ} \mathrm{C}$ in our experimental conditions. Among different nanoparticles suitable for click chemistry [44], or already utilized for coupling with Curc $[30,41]$, we decided to use nanoliposomes, due to their known non-toxic and non-immunogenic features, fully biodegradable and structurally versatile nature [45].

In the present investigation we utilized NL composed of sphingomyelin and cholesterol; different from the formulation already reported [30]. This is a formulation repeatedly utilized in vivo for therapeutic purposes, displaying good circulation times in blood, biocompatibility and resistance to hydrolysis [46].

Starting from this point, we further functionalized Curc-NL with the aim to confer them the ability to cross the BBB. Development of strategies to deliver drugs to the central nervous system is of high importance, because many drug candidates are not able to permeate the BBB. Among all the different ligands proposed for BBB passage (as endogenous and foreign proteins, antibodies, and peptides) [24], here we employed a modified cell penetrating TAT-peptide. TAT protein from HIV-1 or certain small regions of such protein were shown to enter cells when added to the surrounding media [47], and to carry heterologous proteins across the BBB [32]. TAT-peptide used in the present investigation was modified by adding at the $C$-terminal a tryptophan residue for spectrofluorimetric quantitative detection and a cysteine residue for the binding to maleimide function of mal-PEGDSPE lipid incorporated into NL during preparation. Before testing the ability of TAT-Curc-NL to cross the BBB in vitro, we worried whether the functionalization with TAT affected or not the reported ability of Curc-NL [30] to interact with A $\beta$ fibrils. The very high affinity 
Citation: Sancini G, Gregori M, Salvati E, Cambianica I, Re F, et al. (2013) Functionalization with TAT-Peptide Enhances Blood-Brain Barrier Crossing In vitro of Nanoliposomes Carrying a Curcumin-Derivative to Bind Amyloid- $\beta$ Peptide. J Nanomed Nanotechol 4: 171. doi:10.4172/21577439.1000171

of NL towards the peptide, assessed by SPR, confirmed that doublefunctionalized NL retained the ability to bind amyloid $\beta$-peptide.

Successively, we observed that the functionalization of Curc-NL with TAT strongly enhances NL uptake by human brain capillary endothelial cells (hCMEC/D3) in comparison to Curc-NL.

Thus, TAT-Curc-NL increased availability at the neurovascular junction of the cerebral microvasculature forming the BBB will be particularly useful to treat neurological disorders such as $\mathrm{AD}$ and cerebrovascular amyloidosis.

Interestingly, our experiments showed that TAT-Curc-NL were uptaken and clustered in the peri-nuclear region, with reduced cytoplasmic distribution, and that their uptake did not configure an active endocytic mechanism. The mechanism involved in TAT traslocation through cellular membranes is not completely understood, but our observations suggested that the exclusion from endosomal/ lysosomal pathway could facilitate transcellular passage, as already reported [48]. Moreover, it should be underlined that bypassing the endocytic route would be very beneficial when using NP as drug carriers because the putative drug could be degraded within the endosomal/ lysosomal compartment [49].

The ability of TAT-Curc-NL to cross the BBB in vitro was assessed on a transwell system made of cultured hCMEC/D3 cells, displaying characteristics and functionality mimicking the basic features of the $\mathrm{BBB}$. The PE of NL, measured by following two of its components, Sm (adding $\left[{ }^{3} \mathrm{H}\right]-\mathrm{Sm}$ as a tracer) and Curc (followed by MS technique) was much higher for TAT-Curc-NL with respect to Curc-NL, proving the effectiveness of double-functionalized NL to flux across the cellular monolayer. Interestingly, the comparable PE values of $\left[{ }^{3} \mathrm{H}\right]-\mathrm{Sm}$ and Curc-PEG-lipid, suggested that TAT-Curc-NL might be still intact after BBB passage.

\section{Conclusion}

Our results show that TAT-Curc-NL are able to cross an in vitro $\mathrm{BBB}$ model and display an high affinity for $\mathrm{A} \beta$ peptide. Thus, TATCurc-NL might be considered as promising tool for implementing innovative strategies for drug and contrast agent delivery to $\mathrm{AD}$ brain. Further studies aiming at investigate in vivo the pharmacokinetic and biodistribution of TAT-Curc-NL both in healthy and in AD transgenic mice are required.

\section{Acknowledgment}

We thank Pierre-Olivier Couraud for providing the hCMEC/D3. The research leading to these results has received funding from the European Community's Seventh Framework Programme (FP7/2007-2013) under Grant Agreement No. 212043 (NAD).

\section{References}

1. Selkoe DJ (2001) Alzheimer's disease: genes, proteins, and therapy. Physiol Rev 81: 741-766.

2. Mangialasche F, Solomon A, Winblad B, Mecocci P, Kivipelto M (2010) Alzheimer's disease: clinical trials and drug development. Lancet Neurol 9: 702-716

3. Hampel H, Prvulovic D, Teipel S, Jessen F, Luckhaus C, et al. (2011) The future of Alzheimer's disease: the next 10 years. Prog Neurobiol 95: 718-728.

4. Selkoe DJ (2001) Alzheimer's disease results from the cerebral accumulation and cytotoxicity of amyloid beta-protein. J Alzheimers Dis 3: 75-80.

5. Chételat G, Villemagne VL, Pike KE, Ellis KA, Ames D, et al. (2012) Relationship between memory performance and $ß$-amyloid deposition at different stages of Alzheimer's disease. Neurodegener Dis 10: 141-144.
6. Walsh DM, Teplow DB (2012) Alzheimer's disease and the amyloid $\beta$-protein Prog Mol Biol Transl Sci 107: 101-124.

7. Bush Al (2002) Metal complexing agents as therapies for Alzheimer's disease Neurobiol Aging 23: 1031-1038

8. Pollack SJ, Sadler II, Hawtin SR, Tailor VJ, Shearman MS (1995) Sulfonated dyes attenuate the toxic effects of beta-amyloid in a structure-specific fashion. Neurosci Lett 197: 211-214.

9. Sabaté R, Estelrich J (2005) Stimulatory and inhibitory effects of alkyl bromide surfactants on beta-amyloid fibrillogenesis. Langmuir 21: 6944-6949.

10. Re F, Airoldi C, Zona C, Masserini M, La Ferla B, et al. (2010) Beta amyloid aggregation inhibitors: small molecules as candidate drugs for therapy of Alzheimer's disease. Curr Med Chem 17: 2990-3006.

11. Goel A, Kunnumakkara AB, Aggarwal BB (2008) Curcumin as "Curecumin": from kitchen to clinic. Biochem Pharmacol 75: 787-809.

12. Anand $P$, Thomas SG, Kunnumakkara AB, Sundaram $C$, Harikumar KB, et al. (2008) Biological activities of curcumin and its analogues (Congeners) made by man and Mother Nature. Biochem Pharmacol 76: 1590-1611.

13. Kelloff GJ, Crowell JA, Steele VE, Lubet RA, Malone WA, et al. (2000) Progress in cancer chemoprevention: development of diet-derived chemopreventive agents. J Nutr 130: 467S-471S

14. Ono K, Hasegawa K, Naiki H, Yamada M (2004) Curcumin has potent antiamyloidogenic effects for Alzheimer's beta-amyloid fibrils in vitro. J Neurosci Res 75: 742-750.

15. Hong HS, Rana S, Barrigan L, Shi A, Zhang Y, et al. (2009) Inhibition of Alzheimer's amyloid toxicity with a tricyclic pyrone molecule in vitro and in vivo. J Neurochem 108: 1097-1108.

16. Kumaraswamy P, Sethuraman S, Krishnan UM (2013) Mechanistic Insights of Curcumin Interactions with the Core-Recognition Motif of $\beta$-Amyloid Peptide. J Agric Food Chem

17. Garcia-Alloza M, Borrelli LA, Rozkalne A, Hyman BT, Bacskai BJ (2007) Curcumin labels amyloid pathology in vivo, disrupts existing plaques, and partially restores distorted neurites in an Alzheimer mouse model. J Neurochem 102: 1095-1104.

18. Yang F, Lim GP, Begum AN, Ubeda OJ, Simmons MR, et al. (2005) Curcumin inhibits formation of amyloid beta oligomers and fibrils, binds plaques, and reduces amyloid in vivo. J Biol Chem 280: 5892-5901.

19. Wang YJ, Pan MH, Cheng AL, Lin LI, Ho YS, et al. (1997) Stability of curcumin in buffer solutions and characterization of its degradation products. J Pharm Biomed Anal 15: 1867-1876.

20. Sharma RA, Gescher AJ, Steward WP (2005) Curcumin: the story so far. Eu J Cancer 41: 1955-1968.

21. Ray B, Bisht S, Maitra A, Maitra A, Lahiri DK (2011) Neuroprotective and neurorescue effects of a novel polymeric nanoparticle formulation of curcumin (NanoCurc ${ }^{\mathrm{TM}}$ ) in the neuronal cell culture and animal model: implications for Alzheimer's disease. J Alzheimers Dis 23: 61-77.

22. Patel MM, Goyal BR, Bhadada SV, Bhatt JS, Amin AF (2009) Getting into the brain: approaches to enhance brain drug delivery. CNS Drugs 23: 35-58.

23. Craparo EF, Bondì ML, Pitarresi G, Cavallaro G (2011) Nanoparticulate systems for drug delivery and targeting to the central nervous system. CNS Neurosci Ther 17: 670-677.

24. Begley DJ (2004) Delivery of therapeutic agents to the central nervous system: the problems and the possibilities. Pharmacol Ther 104: 29-45.

25. Persidsky Y, Ramirez SH, Haorah J, Kanmogne GD (2006) Blood-brain barrier: structural components and function under physiologic and pathologic conditions. J Neuroimmune Pharmacol 1: 223-236.

26. Brambilla D, Le Droumaguet B, Nicolas J, Hashemi SH, Wu LP, et al. (2011) Nanotechnologies for Alzheimer's disease: diagnosis, therapy, and safety issues. Nanomedicine 7: 521-540.

27. Re F, Gregori M, Masserini M (2012) Nanotechnology for neurodegenerative disorders. Nanomedicine 8 Suppl 1: S51-S58

28. Sahni JK, Doggui S, Ali J, Baboota S, Dao L, et al. (2011) Neurotherapeutic applications of nanoparticles in Alzheimer's disease. J Control Release 152 208-231. 
Citation: Sancini G, Gregori M, Salvati E, Cambianica I, Re F, et al. (2013) Functionalization with TAT-Peptide Enhances Blood-Brain Barrier Crossing In vitro of Nanoliposomes Carrying a Curcumin-Derivative to Bind Amyloid- $\beta$ Peptide. J Nanomed Nanotechol 4: 171. doi:10.4172/21577439.1000171

29. Roney C, Kulkarni P, Arora V, Antich P, Bonte F, et al. (2005) Targeted nanoparticles for drug delivery through the blood-brain barrier for Alzheimer's disease. J Control Release 108: 193-214.

30. Mourtas S, Canovi M, Zona C, Aurilia D, Niarakis A, et al. (2011) Curcumindecorated nanoliposomes with very high affinity for amyloid- $\beta 1-42$ peptide. Biomaterials 32: 1635-1645.

31. Airoldi C, Zona C, Sironi E, Colombo L, Messa M, et al. (2010) Curcumin derivatives as new ligands of $A \beta$ peptides. J Biotechnol 156: 317-324.

32. Schwarze SR, Ho A, Vocero-Akbani A, Dowdy SF (1999) In vivo protein transduction: delivery of a biologically active protein into the mouse. Science 285: 1569-1572.

33. Qin Y, Chen H, Yuan W, Kuai R, Zhang Q, et al. (2011) Liposome formulated with TAT-modified cholesterol for enhancing the brain delivery. Int J Pharm 419: 85-95.

34. Frankel AD, Pabo CO (1988) Cellular uptake of the tat protein from human immunodeficiency virus. Cell 55: 1189-1193.

35. Wadia JS, Stan RV, Dowdy SF (2004) Transducible TAT-HA fusogenic peptide enhances escape of TAT-fusion proteins after lipid raft macropinocytosis. Nat Med 10: 310-315.

36. Torchilin VP, Rammohan R, Weissig V, Levchenko TS (2001) TAT peptide on the surface of liposomes affords their efficient intracellular delivery even at low temperature and in the presence of metabolic inhibitors. Proc Natl Acad Sci U S A 98: 8786-8791.

37. Priyadarsini KI (2009) Photophysics, photochemistry and photobiology of curcumin: Studies from organic solutions, bio-mimetics and living cells. J Photoch Photobio C 10: 81-95.

38. Gobbi M, Re F, Canovi M, Beeg M, Gregori M, et al. (2010) Lipid-based nanoparticles with high binding affinity for amyloid-beta1-42 peptide. Biomaterials 31: 6519-6529.
39. Stewart JC (1980) Colorimetric determination of phospholipids with ammonium ferrothiocyanate. Anal Biochem 104: 10-14.

40. Re F, Cambianica I, Zona C, Sesana S, Gregori M, et al. (2011) Functionalization of liposomes with ApoE-derived peptides at different density affects cellular uptake and drug transport across a blood-brain barrier model. Nanomedicine 7: 551-559.

41. Le Droumaguet B, Nicolas J, Brambilla D, Mura S, Maksimenko A, et al. (2012) Versatile and efficient targeting using a single nanoparticulate platform application to cancer and Alzheimer's disease. ACS Nano 6: 5866-5879.

42. Re F, Cambianica I, Sesana S, Salvati E, Cagnotto A, et al. (2010) Functionalization with ApoE-derived peptides enhances the interaction with brain capillary endothelial cells of nanoliposomes binding amyloid-beta peptide. J Biotechnol 156: 341-346.

43. Re F, Gregori M, Masserini M (2012) Nanotechnology for neurodegenerative disorders. Maturitas 73: 45-51.

44. Li N, Binder WH (2011) Click-chemistry for nanoparticle-modification. J Mater Chem 21: 16717-16734.

45. Torchilin VP (2005) Recent advances with liposomes as pharmaceutical carriers. Nat Rev Drug Discov 4: 145-160.

46. Webb MS, Harasym TO, Masin D, Bally MB, Mayer LD (1995) Sphingomyelincholesterol liposomes significantly enhance the pharmacokinetic and therapeutic properties of vincristine in murine and human tumour models. $\mathrm{Br}$ J Cancer 72: 896-904.

47. Green M, Loewenstein PM (1988) Autonomous functional domains of chemically synthesized human immunodeficiency virus tat trans-activator protein. Cell 55: 1179-1188.

48. Fretz MM, Storm G (2010) TAT-peptide modified liposomes: preparation, characterization, and cellular interaction. Methods Mol Biol 605: 349-359.

49. Torchilin VP (2007) Targeted pharmaceutical nanocarriers for cancer therapy and imaging. AAPS J 9: E128-147. 